\title{
Correction to: Surface Functionalization of Polymers
}

\author{
Deval Prasad Bhattarai, Pashupati Pokharel, and Dequan Xiao
}

\section{Correction to:}

Chapter 2 in: T. J. Gutiérrez (ed.), Reactive and Functional Polymers Volume Four, https://doi.org/10.1007/978-3-030-52052-6_2

The country in Dr. Deval Prasad Bhattarai affiliation is incorrectly mentioned as "India". However, the affiliation is corrected to Department of Chemistry, Amrit Campus, Tribhuvan University, Kathmandu, Nepal. 